Article

\title{
Thermal Transport on Graphene-Based Thin Films Prepared by Chemical Exfoliations from Carbon Nanotubes and Graphite Powders
}

\author{
Jian-De Xie *, Si-Yong Gu ${ }^{(D)}$ and Houan Zhang \\ Fujian Provincial Key Laboratory of Functional Materials and Applications, Institute of Material Preparation \\ and Applied Technology, School of Materials Science and Engineering, Xiamen University of Technology, \\ Xiamen 361024, China; gu-siyong@163.com (S.-Y.G.); ha_zhang@163.com (H.Z.) \\ * Correspondence: 2017000006@xmut.edu.cn; Tel.: +86-592-629-1328
}

Received: 13 July 2017; Accepted: 31 August 2017; Published: 3 September 2017

\begin{abstract}
Thermal conductivities ( $k$ ) of different graphene nanosheet (GN)-based heat sinks are investigated within the temperature range of 323-423 K. One- and two-step modified Hummers' methods are adopted to chemically exfoliate GNs from two kinds of carbon precursors: carbon nanotubes (CNTs) and graphite powders. The two-step method offers an improved exfoliation level of GN products, especially for the CNT precursor. Experimental results show that the GN-based heat sink-exfoliated from graphite powders after the two-step approach-delivers an enhanced $k$ value to $2507 \mathrm{~W} / \mathrm{m} \mathrm{K}$ at $323 \mathrm{~K}$, as compared to the others. The $k$ value is found to be a decreasing function of the porosity of the heat sink, revealing the importance of solid/void fraction (i.e., volumetric heat capacity). The improved thermal efficiency mainly originates from the long phonon mean free path and the low void fraction of GN-based heat sinks, thus inducing highly efficient thermal transport in the GN framework.
\end{abstract}

Keywords: graphene-based thin films; heat sink; thermal conductivity; carbon nanotubes; chemical exfoliation; graphite powders

\section{Introduction}

Recently, one- and two-dimensional carbon nanostructures-especially carbon nanotubes (CNTs) and graphene nanosheets (GNs) - have attracted a great deal of scientific and technological attention, owing to their extraordinary electrical and thermal properties, exhibiting their applicable feasibility [1,2]. Both CNTs and GNs have been demonstrated to display high theoretical thermal conductivities $(k)$, thus heralding beneficial applications in heat exchangers and thermal management systems. It is generally recognized that low-temperature operation and efficient heat dissipation are key factors in determining the lifetime and the speed in current electronic and photonic devices $[1,3]$. Industrially, the most commonly-used materials for heat sinks are still $\mathrm{Al}$ and $\mathrm{Cu}$, which possess lower $k$ values and higher true densities, as compared to carbonaceous materials. Hence, there is torpid progress on the thermal management of interfacial solid surfaces, thus hampering the miniaturization of electronic and photonic devices [4]. To resolve the above problems, one strategy is to adopt nanostructural carbon materials (e.g., GNs) as heat sinks, capable of conducting heat efficiently and thus preventing structural damage to electronic components [5].

It has been reported that single-layer GNs possess a high theoretical $k$ value of 4840-5300 W/m K [6-9], motivating many scientists and researchers to consider GN-based heat sinks for nanoscale device application. However, an obvious gap exists between the actual $k$ values of GN-based layers or heat sinks and the theoretical ones. As to the nano-structured carbons, the $k$ values reported thus far include aligned functionalized multilayer $\mathrm{GN}$ architecture $(112 \mathrm{~W} / \mathrm{m} \mathrm{K}$ at 
298 K) [1], three-dimensional CNT/GN framework (1991 W/m K at $323 \mathrm{~K}$ ) [2], graphene nanoribbons (2300 W/m K at $300 \mathrm{~K}$ ) [7], GN-Cu-GN heterogeneous films (373 W/m K at $300 \mathrm{~K}$ ) [10], and N-doped GN/Cu composite film ( $543 \mathrm{~W} / \mathrm{m} \mathrm{K}$ ) [11]. The major gap can be attributed to the fact that the efficiency of thermal transport is mainly influenced by the presence of topological defects, surface roughness, surface heterogeneity, and porosity in carbon-based heat sinks [7]. Among the properties of carbon-based materials, the porosity may play a crucial role in affecting the thermal conduction in the heat sinks if the pores are full of air, since air has a very low $k$ value $(\sim 0.024 \mathrm{~W} / \mathrm{m} \mathrm{K}$ at ambient temperature). However, few reports have discussed the relationship between the porosity and the $k$ values in GN-based heat sinks.

Accordingly, this work aims to explore the influence of porosity on the efficiency of thermal transport in GNs. An efficient Hummers' method was adopted to chemically exfoliate graphene oxide (GO) sheets from two kinds of carbon precursors (i.e., CNTs and graphite powders). To ensure the completion of chemical exfoliation, the modified Hummers' approach was carried out twice, and the as-prepared GO sheets were thermally reduced in an $\mathrm{H}_{2}$-containing atmosphere. The thermal transport properties of GN-based heat sinks were systematically investigated within a wide temperature range (323-423 K), demonstrating the feasibility of GN-based heat sinks for practical applications.

\section{Materials and Methods}

Compared to the original method of Hummers [12], this work made some modifications in oxidizing agent concentration, oxidation period, and ratio of precursor to oxidation agent to prepare GO sheets, as reported previously $[13,14]$. Two types of carbon precursors-CNTs (AzTrong Inc., Hsinchu, Taiwan) and graphite powders (Taiwan Maxwave Co., Taoyuan, Taiwan)—were of commercial origin. The multi-layered CNT product was grown by chemical vapor deposition method, using Ni-based nanoparticles and ethylene as catalyst and carbon source, respectively. The graphite powder (purity: $99.5 \%$, average particle size: $3 \mu \mathrm{m}$ ) employed here widely serves as a conducting agent for Li-ion batteries. The one-step method of Hummers for producing GO sheets can be briefly described in the following four steps. In step (i), $5 \mathrm{~g}$ carbon powders (i.e., CNTs and graphite powders) were put into $115 \mathrm{~mL}$ concentrated $\mathrm{H}_{2} \mathrm{SO}_{4}$ and well dispersed in an ice bath for $1.5 \mathrm{~h}$. Afterwards, $15 \mathrm{~g} \mathrm{KMnO}_{4}$ was slowly added into the slurry within $1 \mathrm{~h}$ while keeping the temperature less than $5{ }^{\circ} \mathrm{C}$. In step (ii), the slurry was stirred by using a magnetic bar and then heated to $35^{\circ} \mathrm{C}$. Next, $200 \mathrm{~mL}$ distilled water was mixed well with the slurry. The temperature of the slurry was gradually increased to $95{ }^{\circ} \mathrm{C}$ and then kept at that temperature for $0.5 \mathrm{~h}$. The purpose of pouring distilled water into the carbon slurry is to dilute the sulfuric acid, preventing excessive gas generated from the strong chemical reaction. We aimed to prevent a dramatic increase in operating temperature, avoiding the hazard potential of sudden boiling. Moreover, fast exfoliation induced a re-stacking of graphene sheets without diluting the strong oxidizers, based on our in-house study. In step (iii), the chemical oxidation was terminated by adding $500 \mathrm{~mL}$ of $\mathrm{H}_{2} \mathrm{O}_{2}$ solution. The addition of $\mathrm{H}_{2} \mathrm{O}_{2}$ was able to remove the residual potassium permanganate and manganese dioxide, leading to an increase in reactive area for the implantation of surface functionalities during the chemical oxidation process. In step (iv), one thermal reduction process was conducted at $400{ }^{\circ} \mathrm{C}$ under 5 vol $\% \mathrm{H}_{2}$ atmosphere in a horizontal furnace. As for the two-step Hummers' method, step (ii) was repeated once again to reinforce the oxidation extent on the carbon samples (i.e., the second replacement of $\mathrm{MnO}_{3}{ }^{+}$ions).

The as-prepared GN nanostructures were observed by field-emission scanning electron microscopy (FE-SEM; JSM-6701F, JEOL, Kyoto, Japan) and transmission electron microscopy (TEM; JEM-2100, JEOL). An X-ray diffraction (XRD; Labx XRD-6000, Shimadzu, Kyoto, Japan) spectroscope equipped with $\mathrm{Cu}-\mathrm{K} \alpha$ radiation emitter was used to characterize the crystallinity of GN samples. Fourier transformed infrared (FT-IR) spectroscopy was adopted to analyze oxygen functionalities of GN samples. The FT-IR spectra were obtained using a Nicolet Avatar 360 FT-IR spectrometer (SpectraLab Scientific Inc., Markham, ON, Canada). For each sample, 32 scans in the spectral ranges 
were recorded with a resolution of $4 \mathrm{~cm}^{-1}$. The crystalline structure of GN powders was characterized by using Raman spectroscopy (Micro-Raman spectrometer, Renishaw, Gloucestershire, UK).

For the measurement of $k$ values, the GN-based heat sinks could be prepared per the following description. First, a slurry-coating technique was adopted to prepare GN-based layers onto $\mathrm{Cu}$ foil. Herein, all GN samples were uniformly mixed with a binder (poly-vinylidenefluoride) with the weight ratio of 80:20 in N-methyl pyrrolidinone (NMP) solvent to form the graphene slurries. Second, the graphene slurries were well blended with a three-dimensional mixer by using zirconia balls for $15 \mathrm{~min}$. The as-prepared slurries were carefully pasted on the foil substrates (thickness: $\sim 10 \mu \mathrm{m}$ ) with a doctor blade, followed by evaporating the solvent (i.e., NMP) with a blow dryer. The as-prepared graphene layers with an area of $2 \times 5 \mathrm{~cm}^{2}$ were then dried at $120^{\circ} \mathrm{C}$ in a vacuum oven overnight. Finally, the electrode sheets were carefully pressed under a pressure of approximately $193.6 \mathrm{~atm}$. The apparent density ( $\left.\varrho_{\text {app }}\right)$ of heat sinks was determined by the ratio of the mass to a given volume. For the determination, the heat sink was with an area of $2 \times 5 \mathrm{~cm}^{2}$ and its real thickness was measured by optical microscope. The $\varrho_{\text {app }}$ value was thus determined by using the ratio of the weight to the measured volume. For accuracy, three pieces of heat sinks were used to get their average readings. One test platform system for determining the $k$ value of the heat sinks was reported in previous studies [2,4].

High-purity (i.e., 99.95\%) Cu plates with different thicknesses served as the reference material to calibrate the accuracy of $k$ values. The calibration curves of operating temperature against heat transfer from an electrical resistance heater could be obtained. The calibration temperatures ranged from 323 to $423^{\circ} \mathrm{C}$, appropriate for the thermal management system for chips in consumer electronics. To confirm the accuracy, $\mathrm{Al}$ foil was used as the other reference to reveal the small deviations $(<2.0 \%)$ at different temperatures (e.g., the $k$ value of $\mathrm{Al}$ : ca. $237 \mathrm{~W} / \mathrm{m} \mathrm{K}$ at ambient temperature).

All GN-based heat sinks were insulated with heat-preservation cotton to avoid any heat dissipation. The temperature reading was monitored and recorded by using an array of five thermocouples (K-type, accuracy: $0.1^{\circ} \mathrm{C}$ ): one was located at the heater, three at the heat sink, and one for measuring the ambient temperature. The apparent $k$ values of the heat sink could be determined by using Fourier's law, based on one-dimensional heat conduction [15]. The $k$ values were determined by comparing the temperature drops across the heat sink, followed by the calculations of Fourier's law. Herein, each reading was averaged from the $k$ values at different locations of heat sinks.

\section{Results and Discussion}

The as-prepared GN samples were designated as two series: GN-CNT1 and GN-CNT2 (precursor: $\mathrm{CNT}, 1$ and 2: one- and two-step chemical exfoliation) and GN-G1 and GN-G2 (precursor: graphite powders, 1 and 2: one- and two-step chemical exfoliation). Figure 1a,b show FE-SEM images of pristine CNTs and graphite powders, respectively. It can be seen that the CNTs look like coiled-type tubes with an average diameter of 30-50 nm, while the pristine graphite powders show a typical layer-stacking morphology. High-resolution transmission electron microscopy (HR-TEM) micrographs for GN samples prepared by the one- and two-step Hummers' method from CNTs and graphite powders are illustrated in Figure 1c-f, showing different morphologies. Apparently, the one-step Hummers' route cannot completely exfoliate GNs from CNTs and graphite powders. As observed from Figure 1c,d, both pristine CNTs and graphite powders basically keep their original shapes (i.e., tubular and layer-stacking shape). Regarding the two-step approach, the GN-CNT2 sample looks like a broken graphene layer, in which the tubular-type structure was unzipped through the two-step exfoliation route. The GN-G2 sample seems like a transparent and soft silk with few layers, resulting from the full chemical exfoliation. Based on the observation, the two-step chemical procedure is capable of improving the exfoliation level of GN samples for both carbon precursors.

XRD was adopted to further characterize the crystalline structure of GN samples. Figure 2 depicts typical XRD patterns of GN samples, in which both pristine CNTs and graphite powders possess high crystallinity of graphite lattices. An obvious (002) diffraction peak at $26.2-26.4^{\circ}$ can be seen; 
i.e., the $d_{002}$-interlayer spacing distance: $3.41 \AA$ (CNTs) and $3.36 \AA$ (graphite powders). The (002) diffraction peaks tend to shift and broaden after the one- and two-step chemical exfoliation process, demonstrating the presence of GNs [4]. This reflects an obvious increase in the interlayer distance between each graphene layer. Compared to the original values, the $d_{002}$-interlayer spacing distances are up to $3.82 \AA$ (GN-G1), $3.85 \AA$ (GN-G2), $3.43 \AA$ (GN-CNT1), and $3.46 \AA$ (GN-CNT2). This finding implies that the CNT precursor shows better chemical resistance than graphite powder. This possibly originates from one-dimensional tubular structure, in which chemical oxidants (e.g., $\mathrm{SO}_{4}{ }^{2-}$ ions) tend to attack and then oxidize both opened ends of CNT during the chemical exfoliation process. To sum up, the GNs prepared from graphite powders can achieve an efficient exfoliation level when compared to the GNs from CNT precursor.
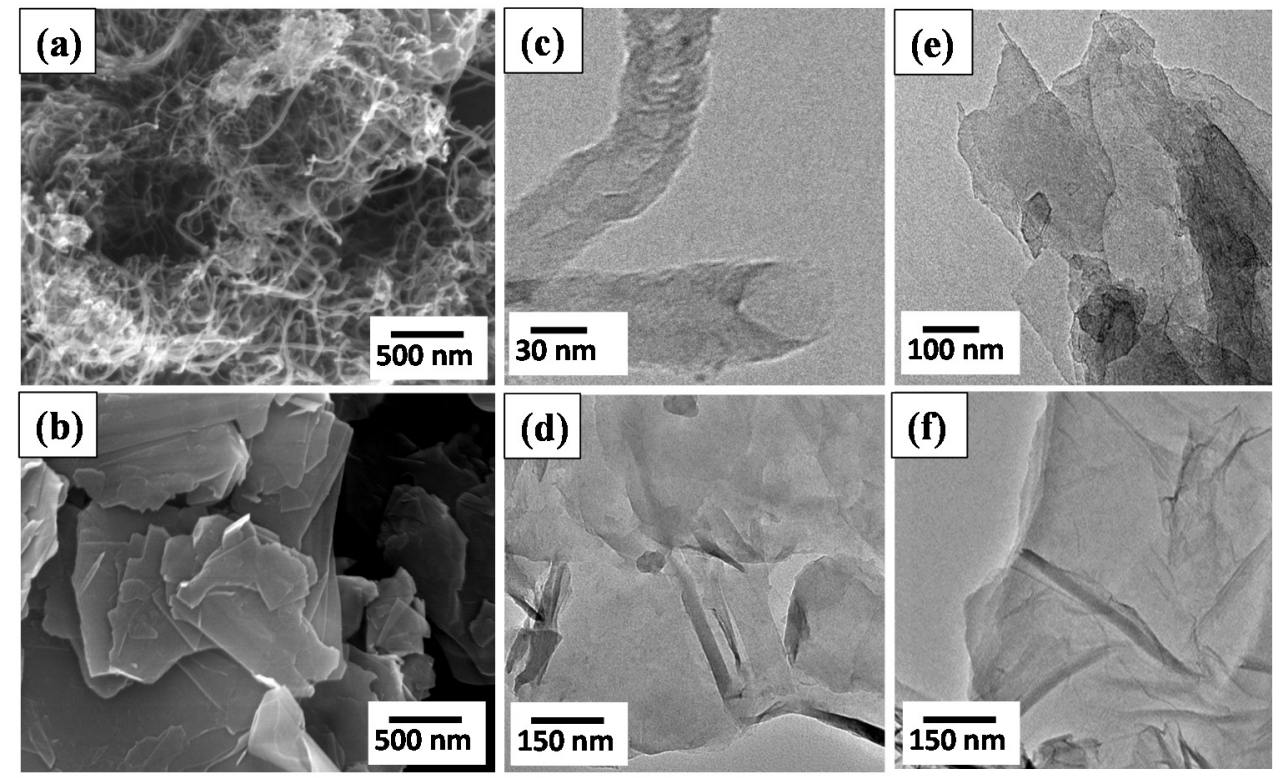

Figure 1. Field-emission SEM (FE-SEM) images of (a) carbon nanotubes (CNTs) and (b) graphite powders. High-resolution transmission electron microscopy (HR-TEM) micrographs of (c) Graphene nanosheet (GN)-CNT1, (d) GN-G1, (e) GN-CNT2, and (f) GN-G2. These images clarify the influence of carbon precursor and exfoliation number on the structral change of as-prepared GN samples. Suffixes 1 and 2 on CNT1/2 and G1/2 refer to one- and two-step chemical exfoliation.

FT-IR spectroscopy was adopted to analyze the distribution of oxygen functional groups on different GN samples, as shown in the Supporting Information (see Figure S1a). The FT-IR spectra reveal the presence of $\mathrm{C}=\mathrm{O}$ and $\mathrm{C}-\mathrm{O}$ groups centered at their specific wavenumbers [16]. It is worth noting that both GN-G1 and GN-CNT1 samples exhibit stronger intensity of transmittance bands compared to the others. This reflects that the first-step chemical oxidation efficiently facilitates the oxidation level of graphene samples, whereas the second-step oxidation-followed by thermal reduction-is capable of stripping oxygen functionalities from basal plane or the edge of graphene samples. Thus, both GN-G2 and GN-CNT2 samples possess a low oxidation extent. As shown in Figure S1b, typical Raman spectra show two characteristic peaks occurring at $1350 \mathrm{~cm}^{-1}$ and $1580 \mathrm{~cm}^{-1}$, corresponding to the $D$ and $G$ bands, respectively. The intensity ratio of $D$ to $G$ band (i.e., $I_{\mathrm{D}} / I_{\mathrm{G}}$ ) serves as a key factor in determining the graphitization degree of carbons, in which the $D$ and $G$ bands indicate the defects or structural disorder and the stacking of the graphite hexagon network plane [17,18]. As compared to the treated GN samples, the pristine $\mathrm{G}$ and CNT samples have lower $I_{\mathrm{D}} / I_{\mathrm{G}}$ ratios -0.33 and 0.51 , respectively. The $I_{\mathrm{D}} / I_{\mathrm{G}}$ ratios of $\mathrm{GN}-\mathrm{G} 1$ and $\mathrm{GN}-\mathrm{G} 2$ samples are approximately 1.18-1.21, whereas the $I_{\mathrm{D}} / I_{\mathrm{G}}$ ratios for both GN-CNT1 and GN-CNT2 samples are up to 1.29-1.45. This increased $I_{\mathrm{D}} / I_{\mathrm{G}}$ ratio can be attributed to the presence of lattice distortion (e.g., 
$\mathrm{sp}^{3} / \mathrm{sp}^{2}$ ratio) and surface functionalities (e.g., $\mathrm{C}-\mathrm{OOH}$ and $\mathrm{C}=\mathrm{O}$ groups) that are decorated in the stacking order of graphite lattice, presumably due to the residual oxygen functionalities after thermal reduction process.

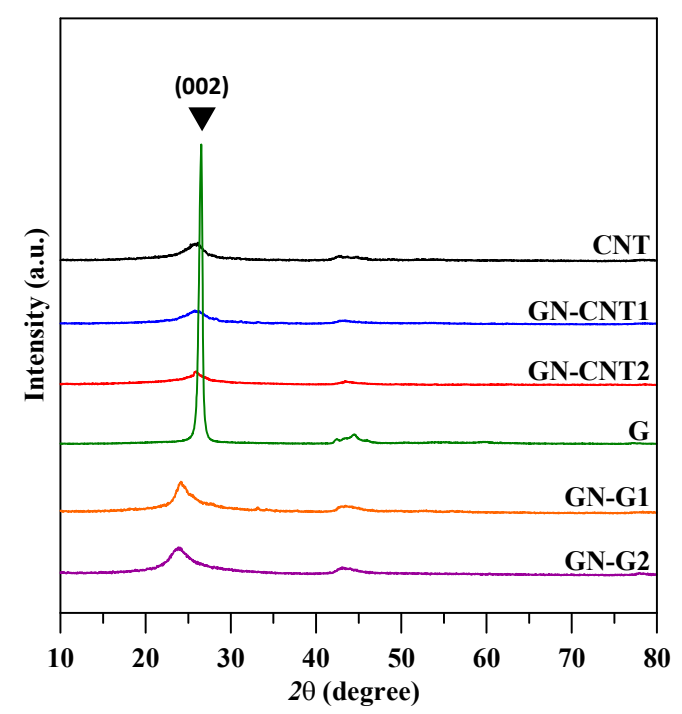

Figure 2. Typical XRD patterns of different GN samples, in which both pristine CNT and graphite (G) powders possess high crystallinity of graphite lattices. Herein, the $d_{002}$-interlayer spacing distances are up to $3.82 \AA$ (GN-G1), $3.85 \AA$ (GN-G2), $3.43 \AA$ (GN-CNT1), and $3.46 \AA$ (GN-CNT2), as compared to the pristine carbon samples.

The variation of $k$ values of heat sinks with operating temperature $(T)$ is illustrated in Figure 3. This figure clearly indicates that the $k$ value shows a decreasing trend with temperature, revealing that the thermal transport in GN-based heat sink is mainly dominated by phonon-boundary scattering. This is because the relationship between $k$ value and $T$ is inconsistent with $1 / T$ temperature dependence within the temperature range, referring to Umklapp phonon-phonon scattering $[19,20]$. The relation delivers two crucial messages: (i) the GN-based heat sinks prepared from graphite powders deliver better thermal diffusivity than that from CNT precursor, and (ii) the two-step exfoliation route significantly improves the efficiency of thermal transport. The experimental results demonstrate that the GN-G2 sample showed the highest $k$ values as compared to the others, reaching as high as $2,507 \mathrm{~W} / \mathrm{m} \mathrm{K}$ at $323 \mathrm{~K}$. In principle, the $k$ value is proportional to a product, $C_{\mathrm{p}} v l$, where $C_{\mathrm{p}}$ is the heat capacity per unit of volume, $v$ the speed of sound, and $l$ the phonon mean free path [5]. The magnitude of $C_{p}$ is strongly related to the solid/void fraction (i.e., porosity) in heat sink, whereas the $l$ value depends on the phonon scattering from carbon boundaries (i.e., grain size), point defects, and Umklapp processes. A pioneering study has pointed out that the CNT-based heat sink consists of highly-resistive thermal junctions between nanotubes [9], thus, leading to smaller $l$ value than the other heat sinks. As observed from HR-TEM and XRD analyses, the CNTs still maintain one-dimensional tubular structure after the one-step exfoliation route. This could be why the GN-CNT1 heat sink displays the lowest thermal transport capability among the heat sinks. With increasing the extent of exfoliation (i.e., after the two-step exfoliation route), the presence of GNs in the heat sinks beneficially facilitates the heat transport, which has been proved by the GNs with high intrinsic $k$ value. Without any thermal resistive junctions, the $k$ value of two-dimensional nanostructures is primarily a function of large mean free path across the strong $\mathrm{sp}^{2}$ bonds in the networks of carbon atoms [21]. Therefore, the stacking of GN architecture can be taken into account as a conductive framework, offering more thermal diffusion paths for heat dissipation.

To inspect the effect of solid-void fraction, the porosity of GN-based heat sinks was measured by using the formula: $\left(1-\varrho_{\text {app }} / \varrho_{\mathrm{t}}\right) \times 100 \%$ [22], where $\varrho_{\text {app }}$ and $\varrho_{\mathrm{t}}$ represent apparent and true 
density of the heat sinks, respectively. The estimated porosity of GN-based heat sinks has an order as follows: GN-CNT1 $(35.1 \%)>$ GN-CNT2 $(22.0 \%)>$ GN-G1 $(20.7 \%)>$ GN-G2 $(19.9 \%)$. It is worth noting that the GN-CNT1 heat sink is mainly composed of coiled-type tubular structure, allowing a large amount of air to be trapped in the nanostructure. The air pockets in the heat sink would raise the porosity and then lower both the $C_{\mathrm{p}}$ and $l$ values, which is unfavorable for the heat transport in GNs. After the two-step exfoliation process, the porosity of GN-based heat sinks tends to be reduced for both carbon precursors. Figure 4a-d shows cross-sectional FE-SEM images of different GN-based heat sinks. As expected, the GN-CNT1 sample possesses a number of voids and cavities, possibly leading to the formation of air pockets in the heat sink. In contrast, the GN-G2 sample is found to have a dense surface, not allowing air storage. The result of porosity analysis reflects that the exfoliation level on the GNs would effectively improve the density of GN stacking layers, thus reducing the porosity of heat sinks.

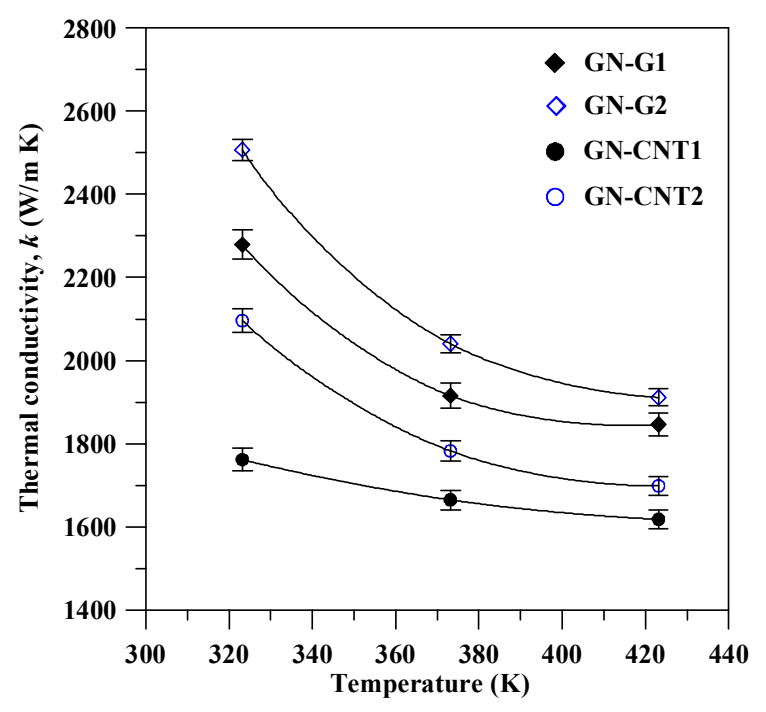

Figure 3. The variation of $k$ values of heat sinks with operating temperature. This figure shows that the $k$ value is a decreasing trend with temperature, revealing that the thermal transport in GN-based heat sink is mainly dominated by phonon-boundary scattering.

The relationship between the porosity and the thermal conduction efficiency is depicted in Figure 5 . As seen in Figure $5 \mathrm{a}$, the GN-CNT1 heat sink displays the largest porosity but the lowest $k$ value among the samples. The $k$ value is illustrated in Figure $5 \mathrm{~b}$ as a decreasing function of the porosity of GN-based heat sinks. This result reveals that the porosity of heat sinks, is strongly correlated to the volumetric $C_{p}$ value, playing an important role in affecting thermal diffusivity. The higher the surface density of GN-based heat sinks, the higher the thermal transport efficiency that can be achieved. However, it is worth noting that the $k$ value is not a linear function of porosity. The improved thermal efficiency mainly originates from high $l$ value and low void fraction of GN-based heat sinks, thus leading to highly efficient thermal transport in the carbon framework. This finding reveals that the efficiency of thermal transport of heat sinks depends not only on the intrinsic thermal properties (e.g., intrinsic $k$ value), but also on the physical and mechanical preparation method (e.g., compressive treatment). Using the same test platform, the GN-G2 sample was found to possess higher $k$ value than the other designs of heat sinks such as CNT/GN hybrid (1991 W/m K at $323 \mathrm{~K}$ ) [2] and $\mathrm{Al}_{2} \mathrm{O}_{3}$-coated graphite $(1128 \mathrm{~W} / \mathrm{m} \mathrm{K}$ at $323 \mathrm{~K})$ [3]. It can be concluded that the selection of carbon materials is the major contributor to the thermal conduction. However, the preparation of heat sinks including chemical composition, rolling process, and compression ratio also is also crucial in affecting the thermal diffusivity. The resultant heat sink used in the present work shows a high compression ratio that may alleviate the effect of $\mathrm{O} / \mathrm{C}$ ratio on the thermal conduction performance. On the basis of 
experimental results, the GNs could serve as a feasible material for high-performance thermal devices, such as heat exchangers and heat sinks for consumer electronic devices or integrated circuit chips. Moreover, the $k$ value of the GN-G2 sample is approximately 6.5 and 10.5 times higher than that of $\mathrm{Cu}$ and $\mathrm{Al}$ foils, respectively. This result demonstrates that the robust design of the GN-G2 sample is superior to that of conventional metal-based heat sinks due to its lightweight, flexibility, and excellent thermal conduction.
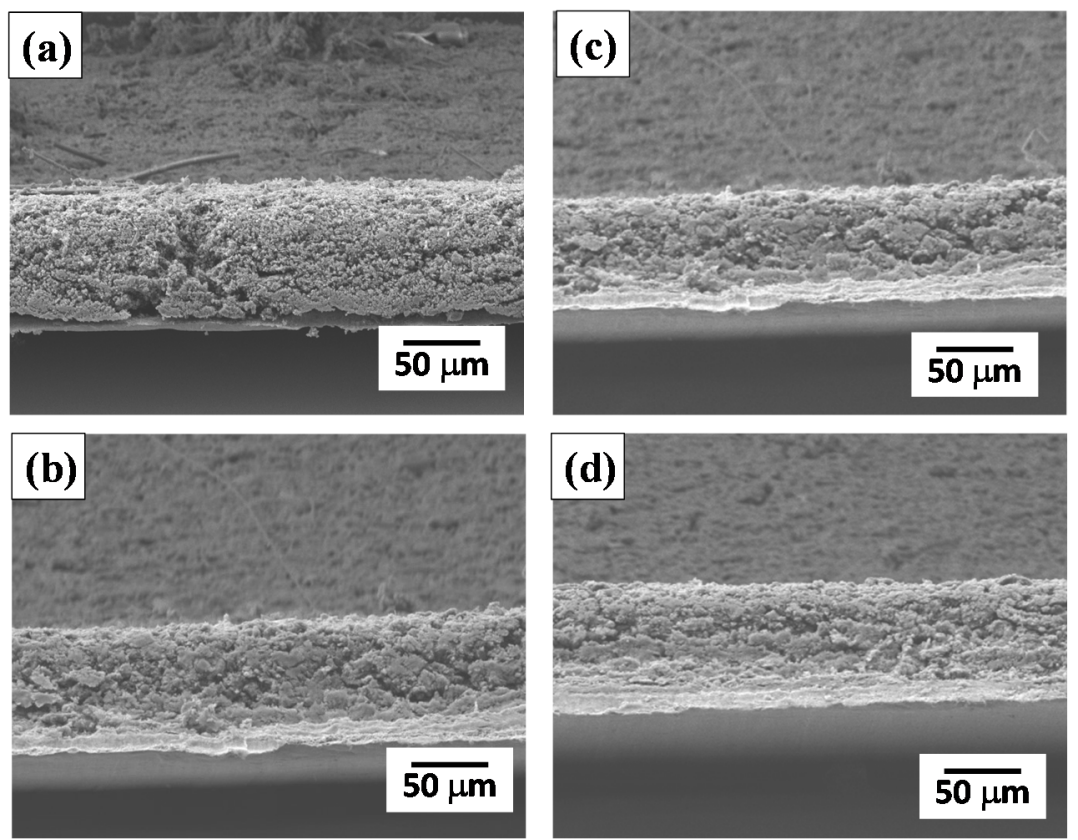

Figure 4. Cross-sectional FE-SEM images of different GN-based heat sinks: (a) GN-CNT1; (b) GN-G1; (c) GN-CNT2; and (d) GN-G2. This reflects that the exfoliation level on as-prepared GNs effectively improves the density of GN stacking layers, thus reducing the porosity of heat sinks.

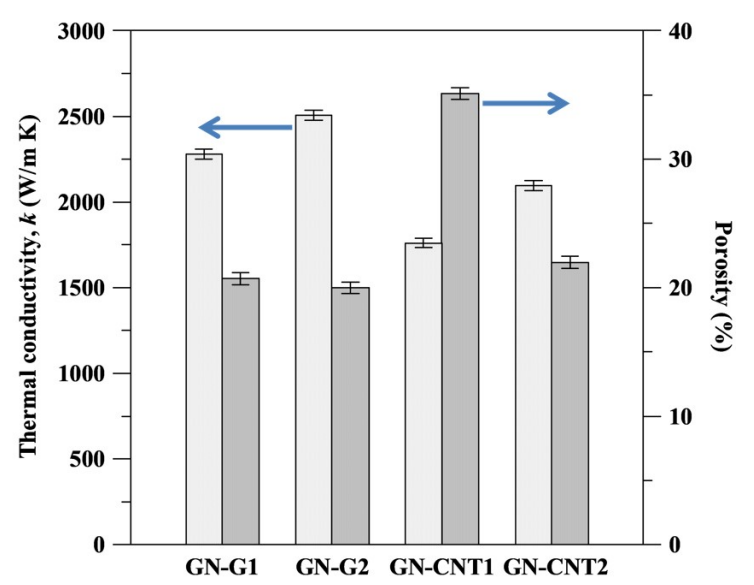

(a)

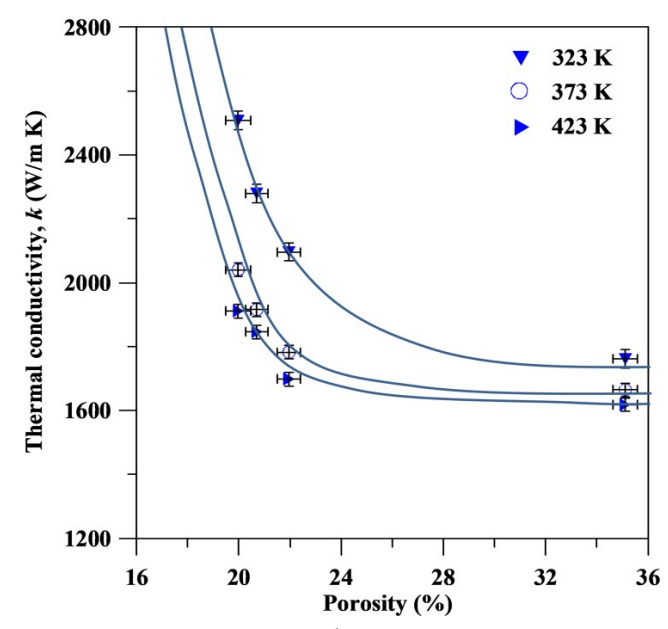

(b)

Figure 5. (a) The relationship between the porosity and the $k$ value and (b) the $k$ value as a decreasing function of the porosity of GN-based heat sinks. This result reveals that the porosity of heat sink is strongly correlated to the volumetric $C_{\mathrm{p}}$ value, playing an important role in affecting thermal diffusivity. 


\section{Conclusions}

We have presented the efficiency of thermal transport in GN-based heat sinks, chemically exfoliated from CNTs and graphite powders using one- and two-step modified Hummers' method, within the temperature region of 323-423 K. The CNT precursor showed strong chemical resistance to chemical oxidizers, whereas the graphite powders could be chemically exfoliated to GN products with high exfoliation extent. The two-step method displayed an improved exfoliation level of GN products-especially for CNT precursor. The GN-based heat sink, exfoliated from graphite powders after the two-step approach could attain the highest $k$ value of $2507 \mathrm{~W} / \mathrm{m} \mathrm{K}$ at $323 \mathrm{~K}$, as compared to the others. The $k$ value as a decreasing function of the porosity of $\mathrm{GN}$-based heat sinks was explored. This result reflected that the porosity of heat sink-strongly correlated to the volumetric $C_{\mathrm{p}}$ value-is one of the crucial factors in determining the thermal diffusivity. The lower the porosity of GN-based heat sinks, the higher the thermal transport efficiency that can be achieved. Since the $k$ value is not a linear function of porosity, its improved value could be attributed to both high $l$ value and low void fraction of GN-based heat sinks, thus leading to highly efficient thermal transport in the carbon framework. As a result, this work delivered a feasible possibility of GN-based films for the future development of heat exchanger and heat sink applications.

Supplementary Materials: The following are available online at http://www.mdpi.com/2079-6412/7/9/138/s1. Figure S1: (a) FT-IR patterns and (b) Raman spectra of different graphene samples. This reflects that the first-step chemical oxidation efficiently facilitates the oxidation level of graphene samples, whereas the second-step oxidation, followed by thermal reduction, is capable of stripping oxygen functionalities from basal plane or edge of graphene samples.

Acknowledgments: The authors are very grateful for the financial support from Xiamen University of Technology.

Author Contributions: Jian-De Xie conceived and designed the experiments; Si-Yong Gu performed the experiments and analyzed the data; Houan Zhang contributed reagents/materials/analysis tools. Jian-De Xie data analysis, article writing and language revision; Si-Yong Gu literature collection, data collection and figures plotting; Houan Zhang data interpretation and discussion.

Conflicts of Interest: The authors declare no conflict of interest.

\section{References}

1. Liang, Q.; Yao, X.; Wang, W.; Liu, Y.; Wong, C.-A. Three-dimensional vertically aligned functionalized multilayer graphene architecture: An approach for graphene-based thermal interfacial materials. ACS Nano 2011, 5, 2392-2401. [CrossRef] [PubMed]

2. Hsieh, C.-T.; Lee, C.-E.; Chen, Y.-F.; Chang, J.-K.; Teng, H. Thermal conductivity from hierarchical heat sinks using carbon nanotubes and graphene nanosheets. Nanoscale 2015, 7, 18663-18670. [CrossRef] [PubMed]

3. Hsieh, C.-T.; Chen, Y.-F.; Lee, C.-E.; Juang, S.-H.; Lin, Z.-W.; Huq, M.M. Enhancing thermal transport efficiency in carbon composites using nanospacers. RSC Adv. 2016, 6, 61351-61356. [CrossRef]

4. Hsieh, C.-T.; Chen, Y.-F.; Lee, C.-E.; Chiang, Y.-M.; Teng, H. Thermal transport in stereo carbon framework using graphite nanospheres and graphene nanosheets. Carbon 2016, 106, 132-141. [CrossRef]

5. Berber, S.; Kwon, Y.K.; Tomanek, D. Unusually high thermal conductivity of carbon nanotubes. Phys. Rev. Lett. 2000, 84, 4613-4616. [CrossRef] [PubMed]

6. Liu, Z.; Guo, Q.; Shi, J.; Zhai, G.; Liu, L. Graphite blocks with high thermal conductivity derived from natural graphite flake. Carbon 2008, 46, 414-421. [CrossRef]

7. Haskins, J.; Kınacı, A.; Sevik, C.; Sevinçli, H.; Cuniberti, G.; Çağın, T. Control of thermal and electronic transport in defect-engineered graphene nanoribbons. ACS Nano 2011, 5, 3779-3787. [CrossRef] [PubMed]

8. Balandin, A.A.; Ghosh, S.; Bao, W.; Calizo, I.; Teweldebrhan, D.; Miao, F.; Lau, C.N. Superior thermal conductivity of single-layer graphene. Nano Lett. 2008, 8, 902-907. [CrossRef] [PubMed]

9. Wang, Q.; Han, X.; Sommers, H.A.; Park, Y.; Joen, C.T.; Jacobi, A. A review on application of carbonaceous materials and carbon matrix composites for heat exchangers and heat sinks. Int. J. Refrigeration 2012, 35, 7-26. [CrossRef]

10. Goli, P.; Ning, H.; Li, X.; Lu, C.; Novoselov, K.S.; Balandin, A.A. Thermal properties of graphene-copper-graphene heterogeneous films. Nano Lett. 2014, 14, 1497-1503. [CrossRef] [PubMed] 
11. Hsieh, C.-C.; Liu, W.-R. Synthesis and characterization of nitrogen-doped graphene nanosheets/copper composite film for thermal dissipation. Carbon 2017, 118, 1-7. [CrossRef]

12. Hummers, W.S., Jr.; Offeman, R.E. Preparation of graphitic oxide. J. Am. Chem. Soc. 1958, 80, 1339. [CrossRef]

13. Hsieh, C.-T.; Hsu, S.-M.; Lin, J.-Y.; Teng, H. Electrochemical capacitors based on graphene oxide sheets using different aqueous electrolytes. J. Phys. Chem. C 2011, 115, 12367-12374. [CrossRef]

14. Hsieh, C.-T.; Yang, B.-H.; Lin, J.-Y. One- and two-dimensional carbon nanomaterials as counter electrodes for dye-sensitized solar cells. Carbon 2011, 49, 3092-3097. [CrossRef]

15. Gong, Q.-M.; Li, Z.; Wang, Y.; Wu, B.; Hang, Z.; Liang, J. The effect of high-temperature annealing on the structure and electrical properties of well-aligned carbon nanotubes. Mater. Res. Bull. 2007, 42, 474-481. [CrossRef]

16. Krishnamoorthy, K.; Veerapandian, M.; Yun, K.; Kim, S.J. The chemical and structural analysis of graphene oxide with different degrees of oxidation. Carbon 2013, 53, 38-49. [CrossRef]

17. Dato, A.; Radmilovic, V.; Lee, Z.; Phillips, J.; Frenklach, M. Substrate-free gas-phase synthesis of graphene sheets. Nano Lett. 2008, 8, 2012-2016. [CrossRef] [PubMed]

18. Guo, H.; Song, L.; Guo, W.; Huang, L.; Yang, D.; Wang, F.; Zuo, Y.; Fan, X.; Liu, Z.; Vajtai, R.; et al. A simple method to synthesize continuous large area nitrogen-doped graphene. Carbon 2012, 50, 4476-4482. [CrossRef]

19. Pop, E.; Mann, D.; Wang, Q.; Goodson, K.; Dai, H. Thermal conductance of an individual single-wall carbon nanotube above room temperature. Nano Lett. 2006, 6, 96-100. [CrossRef] [PubMed]

20. Ziman, J.M. Eletrons and Phonons; Oxford University Press: Oxford, UK, 2001.

21. Warzoha, R.J.; Zhang, D.; Feng, G.; Fleischer, A.S. Engineering interfaces in carbon nanostructured mats for the creation of energy efficient thermal interface materials. Carbon 2013, 61, 441-457. [CrossRef]

22. Jeong, H.-S.; Lee, S.-Y. Closely packed $\mathrm{SiO}_{2}$ nanoparticles poly(viylidene fluoride-hexafluorpropylene) layers-coated polyethylene separator for lithium-ion batteries. J. Power Sources 2011, 196, 6716-6722. [CrossRef]

(C) 2017 by the authors. Licensee MDPI, Basel, Switzerland. This article is an open access article distributed under the terms and conditions of the Creative Commons Attribution (CC BY) license (http:/ / creativecommons.org/licenses/by/4.0/). 